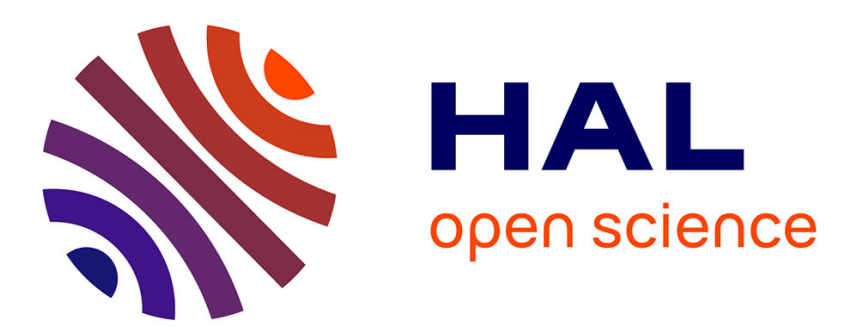

\title{
Three distinct modes in a cold atmospheric pressure plasma jet
}

\author{
J L Walsh, F Iza, N B Janson, V Law, M G Kong
}

\section{To cite this version:}

J L Walsh, F Iza, N B Janson, V Law, M G Kong. Three distinct modes in a cold atmospheric pressure plasma jet. Journal of Physics D: Applied Physics, 2010, 43 (7), pp.75201. 10.1088/00223727/43/7/075201 . hal-00569762

\section{HAL Id: hal-00569762 \\ https://hal.science/hal-00569762}

Submitted on 25 Feb 2011

HAL is a multi-disciplinary open access archive for the deposit and dissemination of scientific research documents, whether they are published or not. The documents may come from teaching and research institutions in France or abroad, or from public or private research centers.
L'archive ouverte pluridisciplinaire HAL, est destinée au dépôt et à la diffusion de documents scientifiques de niveau recherche, publiés ou non, émanant des établissements d'enseignement et de recherche français ou étrangers, des laboratoires publics ou privés. 


\title{
Three distinct modes in a cold atmospheric pressure plasma jet
}

\author{
J. L. Walsh ${ }^{1}$, F. Iza ${ }^{1}$, N. B. Janson ${ }^{2}$, V. Law ${ }^{3}$, and M. G. Kong ${ }^{1 *}$ \\ 1 Department of Electronic and Electrical Engineering, Loughborough University, UK \\ 2 School of Mathematics, Loughborough University, UK \\ 3 National Centre for Plasma Science and Technology, Dublin City University, Ireland
}

\begin{abstract}
Cold atmospheric pressure helium plasma jets are increasingly used in many processing applications, due to a distinct combination of their inherent plasma stability with excellent reaction chemistry often enhanced downstream. Despite their widespread usage, it remains largely unknown whether cold atmospheric plasma jets maintain similar characteristics from breakdown to arcing or whether they possess different operating modes. In addition to their known ability to produce a fast moving train of discrete luminous clusters along the jet length, commonly known as plasma bullets, this paper reports evidence of two additional modes of operation, namely a chaotic mode and a continuous mode in an atmospheric helium plasma jet. Through detailed electrical and optical characterisation, it is shown that immediately following breakdown the plasma jet operates in a deterministic chaotic mode. With increasing input power, the discharge becomes periodic and the jet plasma is found to produce at least one strong plasma bullet every cycle of the applied voltage. Further increase in input power eventually leads to the continuous mode in which excited species are seen to remain within the inter-electrode space throughout the entire cycle of the applied voltage. Transition from the chaotic, through the bullet, to the continuous modes is abrupt and distinct, with each mode having a unique set of operating characteristics. For the bullet mode, direct evidence is presented to demonstrate that the evolution of the plasma jet involves a repeated sequence of generation, collapse and regeneration of the plasma head occurring at locations progressively towards the instantaneous cathode. These offer previously unavailable insight into plasma jet formation mechanisms and the potential of matching plasma jet modes to specific needs of a given processing application.
\end{abstract}

\footnotetext{
* Author to whom correspondence should be addressed; e-mail: m.g.kong@lboro.ac.uk
} 


\section{I ntroduction:}

Cold atmospheric pressure plasma jets have recently commanded much interest [1]-[4]. They offer a chamberless delivery of downstream reaction chemistry, ideal for polymeric surface modification [5][6], bacterial and biomolecule inactivation [7][8], wound healing [9][10], and nano-structure fabrication [11][12]. A distinct characteristic of a jet configuration is its ability to generate stable discharge in a region of inert gas and then to transport the plasma to a separate region of reactive gas for processing applications, thus providing chemical reactivity without compromising plasma stability [13]. This spatial separation of the plasma generation and surface processing regions enables a considerable flexibility in jet designs to vary and control both plasma dynamics and reaction chemistry. Atmospheric plasma jets can be generated in many different gases and using different electrical excitations. These include DC jets employing air and nitrogen [14][15], kHz frequency pulsed and sinusoidal jets [16]-[19], radio frequency (RF) [9][20]-[24] and microwave excited jets [25]-[30]. Depending on jet configuration and electrical excitation, plasma characteristics may differ significantly. Typically DC and microwave jets produce hot dense plasmas and are ideal for treating heat resistive materials. For heat sensitive materials, their gas temperature needs to be reduced drastically. This can be achieved by placing the sample far away from the jet nozzle, employing fast gas flow, generating small plasma volumes and/or using different excitation frequencies. RF plasma sources have lower gas temperature and good plasma stability benefited partly from electron trapping and efficient electron heating at the sheath edges [31][32]. However for treating heat-sensitive materials, it is still desirable to reduce their gas temperature particularly at high RF power. This may be addressed by using pulse-modulated RF excitation [33][34]. At lower frequencies below $100 \mathrm{kHz}$, sub-microsecond pulsed excitation is found to effectively reduce gas temperature [13][19][35]-[38]. In principle, it is possible to use nanosecond pulses and produce room-temperature atmospheric air plasma even at the core of the discharge [37]. The above brief summary highlights that plasma characteristics vary considerably with temporal 
features of electrical excitation, chemical composition and flow rates of the carrier gas, and indeed electrode configurations.

Although the presence of different operating modes (filamentary, Townsend, glow, etc) have been identified in atmospheric pressure dielectric barrier discharges sustained between two parallel electrodes [39][40], it remains at present largely unknown whether the atmospheric plasma jet evolves without any fundamental change in characters from breakdown to arcing. In the context of operation characteristics of atmospheric plasma jets, a major topic of current interest has been plasma bullets, a fast moving train of highly luminous but discrete clusters along the plasma jet when imaged on a nanosecond scale [17]. Several theories of bullet formation have been proposed [19][41]-[44], however to date a definite explanation of the physical mechanisms driving the plasma bullet phenomenon remains elusive. This line of current research would benefit from a more detailed characterization of plasma bullet behavior and indeed from exploring whether the regime of plasma bullets may border with other discharge regimes. The latter issue is directly related to the need for establishing how a given plasma jet may change in its dynamic behaviors from ignition to arcing.

This work is concerned with identification and characterization of operating modes in linear-field [45] atmospheric-pressure kHz helium plasma jets. Based on electrical, optical and imaging characterization, this study will demonstrate the presence of two other modes in addition to the plasma bullet mode, namely a chaotic mode and a continuous mode. The chaotic mode is observed after gas breakdown. With increasing input power, the discharge enters the bullet mode and at high enough input power, the continuous mode is observed. Transition from the chaotic mode, through the bullet, to the continuous mode is abrupt and distinct, with each mode having a unique set of operating characteristics. The aim of this work is to provide a detailed characterisation of the three modes and investigate the underlying physical mechanisms responsible for each mode. From an application perspective, a better understanding of each mode enables a valuable potential of tailoring plasma characteristics to match application requirements. 


\section{Experimental Apparatus and Methods:}

The plasma jet considered in this study is similar to those used in several previous studies [42][45]. Shown in figure 1 , the device consists of a quartz tube measuring $5 \mathrm{~cm}$ in length with a $2 \mathrm{~mm}$ inner diameter and $3 \mathrm{~mm}$ outer diameter. A $1 \mathrm{~cm}$ wide metallic electrode is wrapped tightly around the quartz tube at a distance of $1 \mathrm{~cm}$ from the tube nozzle. A second grounded metallic electrode is placed $1.5 \mathrm{~cm}$ downstream from the nozzle, this can either be dielectrically coated or bare metallic. For this study, the downstream electrode was left bare. An MKS mass flow controller was employed to provide a constant helium flow (99.996\% purity) of 5 standard liters per minute (SLM) through the tube for all cases reported here. Gas flow rate and background gas composition were found to affect the parametric ranges of the three modes. These will be reported in a future publication. A home-built power source, capable of producing a variable amplitude high voltage sinusoid at a fixed frequency of $18 \mathrm{kHz}$ was connected to the wrapped electrode. The electric field set up between the wrapped ring electrode and the downstream ground electrode was largely in the axial direction, parallel to the direction of the carrier gas flow through the quartz tube, i.e. the plasma jet was a linear-field device [45].

For electrical measurements, a Tektronix P6015A 75MHz high voltage probe was connected to the wrapped electrode and a Pearson $28771 \mathrm{~V} / 1 \mathrm{~A} 200 \mathrm{MHz}$ current monitor was inserted between the ground electrode and the grounding point. A Tektronix DPO4104 oscilloscope was employed to record current and voltage signals, with its $1 \mathrm{GHz}$ bandwidth and 10 mega-sample record length making it an ideal choice for recording many cycles of the applied voltage. For the chaotic mode, this enabled confirmation of its non-periodic nature over many cycles. For optical diagnostics, an optical fiber was positioned at $1 \mathrm{~mm}$ below the tube nozzle and fixed radially $5 \mathrm{~mm}$ from the axis of the plasma plume. Emission spectra were obtained using an Andor Shamrock SR-303i spectrometer with a focal length of $303 \mathrm{~mm}$. Detailed spectra were acquired using a 2400 lines/mm grating providing a $0.01 \mathrm{~nm}$ spectral resolution, overview spectra were obtained using a 600 lines $/ \mathrm{mm}$ grating providing a $0.13 \mathrm{~nm}$ spectral resolution. 
Absolute emission intensity calibration was performed with an Ocean Optics LS-1-CAL NIST traceable white light source, providing absolute intensity values from 350-1000nm.

\section{Electrical and I maging Characteristics:}

Figure 2 details current and voltage data for each of the three modes of operation, for which the plasma appearance is diffuse, stable and largely homogenous. The chaotic mode is observed immediately after breakdown, and an increase in the input power eventually leads to the bullet mode and then to the continuous mode. Evidence for the chaotic mode will be detailed below. The role of seed electrons and surface charges, and the dynamic balance between the applied and memory voltages (voltage across the dielectrics) required to reignite the discharge in each excitation cycle is well described in the literature of dielectric barrier discharges [39][46].

A common feature of all three modes is that their current waveform has one distinct peak every positive half-cycle of the applied voltage. For the chaotic mode, the negative half-cycles often have a current peak but this is not always the case, for example the $1^{\text {st }}$ and $4^{\text {th }}$ negative half-cycles in figure 2a. Even for cycles with distinct current peaks in both the positive and negative half-cycles, the magnitude of the current peaks and their occurrence instants vary significantly from one cycle to another. Sometimes two or more small spikes appear on top of one current peak and these usually happen in the positive half-cycles, for example in the $4^{\text {th }}$ and $5^{\text {th }}$ positive half-cycle in figure $2 \mathrm{a}$. The maximum variation in magnitude between any two consecutive current peaks is about $30 \%$. The appearance of the positive current peaks occurs at a mean value of $11.7 \mu \mathrm{s}$ after the previous zero-cross point of the voltage waveform (from negative to positive), with a standard deviation of $3.3 \mu \mathrm{s}$. At $18 \mathrm{kHz}$, one half period is $27.8 \mu \mathrm{s}$ and so the occurrence time of positive current peaks varies about $12 \%$ around its mean value. In this chaotic mode, small variations in the number of seed electrons in the discharge gap and surface charges on the dielectric tube between subsequent cycles result in the ignition of 
plasmas with very different characteristics, i.e. the device displays deterministic chaotic behavior [47]. Although chaotic behavior has been observed in other plasma systems, this is the first report on the chaotic behavior of atmospheric pressure plasma jets.

As the applied voltage is increased sufficiently, plasma dynamics become periodic as shown in the current waveforms in figure $2 \mathrm{~b}$ and $2 \mathrm{c}$. For both the bullet mode and the continuous mode, there is one strong current peak every half-cycle, typical of dielectric barrier discharges [39][46][48]. These strong current peaks are due to the rapid increase in gas conductivity at plasma ignition followed by an accumulation of surface charge acting to reduce the gas voltage and extinguish the discharge [39][46][48]. In figure $2 b$, the current pulses in either positive or negative half-cycles appear consistently at $10 \pm 0.01 \mu$ s after the preceding voltage zero crossing point. Their magnitude changes by less than $0.5 \%$, much smaller than that in the chaotic mode, suggesting an inherent regulation of plasma dynamics from one cycle to the next [46]. In the case of figure $2 c$ with a larger applied voltage, the current peaks become greater in magnitude and broader in duration. In fact, the duration of the current peaks in figure $2 \mathrm{c}$ is comparable to the half-period of the applied voltage, suggesting a continuation of gas discharge from one half-cycle to the next. Current waveforms in figure 2 are very different from one mode to another.

With the evidence of three different modes from figure 2, it is important to characterize the mode transitions. Mode transition is usually captured in the current-voltage characteristics, for example in radio-frequency atmospheric glow discharges [49][50]. However in the chaotic mode, the discharge current varies in its magnitude from one cycle to the other and its temporal characters are different from that of the applied voltage. Therefore it is inappropriate to relate the current magnitude to the voltage magnitude for the chaotic mode. An alternative is to employ the average dissipated power, which evens out the variation in the discharge current and represents a quantifiable measure of the extent of the discharge. This is shown in figure 3 as a function of the applied voltage. Breakdown is seen to occur at an applied voltage of $V_{\mathrm{rms}}=1.34 \mathrm{kV}$, after which the plasma appeared weak to the naked eye and the dissipated 
power was very low. As the applied voltage was increased, the number of cycles where a current pulse was present increased rapidly (data not shown). The increase in the number of gas discharge events and in their intensity is reflected in an almost exponential increase in the dissipated power up to $V_{\mathrm{rms}}=1.73 \mathrm{kV}$ after which the current waveform resembles that shown in figure $2 \mathrm{~b}$. Further increases in the applied voltage result in a broadening of the current pulse combined with a moderate increase in magnitude. Finally, at $V_{\mathrm{rms}}=2.50 \mathrm{kV}$ each current pulse becomes so wide in duration that it merges with the next, indicating a continuous discharge. As the applied voltage is increased further, the discharge current undergoes a rapid growth and this increases power dissipation within the discharge. Figure 3 highlights that the three modes are clearly discernable from measurements of dissipated power; the gradient of each line gives an indication of the electrical characteristics of the discharge. In the bullet mode, a $1 \mathrm{kV}$ increase in the applied voltage results in less than a $1 \mathrm{~W}$ increase in dissipated power; however, in the continuous mode a $1 \mathrm{kV}$ increase in the applied voltage yields a $3 \mathrm{~W}$ increase in dissipated power, indicating a jump in electrical conductivity. In general, mode transition is abrupt and distinct.

To further characterize the three modes, typical power spectral densities (spectra) of the three modes are shown in figure 4 . Each spectrum in figure 4 is obtained as follows: (i) a squared amplitude $\left|F(f)^{2}\right|$ of the Fourier Transform of the current registered during 20 excitation cycles is calculated, where $f$ is the frequency; (ii) $\left|F(f)^{2}\right|$ is then averaged over 15 different realizations of the current; and (iii) the averaged function $\overline{\left|F(f)^{2}\right|}$ is normalized by the duration $T$ of a single realization, where $T=20 / 18 \mathrm{kHz}$. The ideal spectra of periodic processes consist of peaks of zero width (delta-peaks) at multiples of the basic frequency, which in our case is the excitation frequency of $18 \mathrm{kHz}$. Due to the finite observation time, the spectra of the bullet and continuous modes in figure 4 contain peaks of finite width imposed on a continuous background. As the observation time increases, these peaks get sharper and the background lower. On the other hand, the spectra of deterministically chaotic and of random 
processes are continuous functions of frequency [47]. They might contain sharp peaks, but these would be imposed on a continuous, usually smooth, background, which will not disappear if the observation time grows. The spectrum of the chaotic mode shown in figure 4 has these features and is therefore an evidence of the non-periodic nature of the current oscillations in the chaotic mode.

Power spectral density alone, however, is not sufficient to establish the existence of deterministic chaos. An additional evidence would be a phase portrait [47]. In particular, periodic oscillations are represented in the phase space by limit cycles (i.e. closed loops), while chaotic oscillations are represented by chaotic attractors, which are generally a set of points with complex structure and non-integer dimension. In addition to the phase portrait, the Poincaré section helps to reveal the detailed structure of an attractor: a section of a limit cycle is one or several points, while the section of a chaotic attractor is typically a set with selfsimilar structure. If the chaotic attractor is relatively simple, its section looks like a horseshoe. From the mathematical viewpoint, the system under study is a spatially extended system in which currents and voltages depend not only on time but also on space. The full collection of such space-time dependent functions will form the phase space of the system, and the dimension of the system will be infinitely large. It has been shown, however, that many infinite-dimensional dynamical systems can have attractors of finite dimension [51]-[54].

For the present study, we have access to only a single observable, namely, the current at the driving electrode $I(t)$, and investigation based on the full set of state variables of the jet is beyond the scope of this work. Therefore, in order to introduce a phase-space representation of the jet, the technique of embedding is used. This technique is based on a number of mathematical results valid for dynamical systems with attractors [55]-[59]. In particular, we use the possibly simplest delay embedding, within which the state vector at time $t$ in the $m$ dimensional phase space is reconstructed as a vector whose coordinates are the values of the single observable taken at time moments separated by a certain delay $\tau$, namely, $(I(t), I(t+\tau)$, $I(t+2 \tau), \ldots, I(t+(m-1) \tau))$. The number $m$ is the embedding dimension and depends on the 
dimension of the attractor in the original dynamical system [58]. For visualization purposes, here we choose $m=3$. The time delay $\tau$ can be chosen by a variety of methods, but one of the most popular approaches is to calculate the mutual information from the variables $I(t)$ and $I(t+\tau)$ as a function of $\tau$, and to choose its first minimum [60]. The value of $\tau$ obtained by this method was close to $4 \mu \mathrm{s}$ for all datasets and was chosen for the phase space reconstruction.

Figures $5 a, 5 c$, and $5 e$ show three-dimensional phase space reconstructions for the plasma jet operating in the chaotic, bullet and continuous modes, respectively. For each mode, the phase trajectory is shown during several hundred excitation cycles. Whereas figures $5 \mathrm{c}$ and $5 \mathrm{e}$ show limit cycles (i.e. periodic attractors), figure 5a shows a set that does not look similar to a limit cycle, nor to a low-dimensional torus representing a quasiperiodic (i.e. non-chaotic) behavior. We therefore suggest that this is a projection of a chaotic attractor into a threedimensional space. However, to obtain a further evidence of that we need a Poincaré section. For systems perturbed periodically it is convenient to introduce a stroboscopic section, which consists of the points of the (possibly reconstructed) phase trajectory taken in a period of external forcing. The stroboscopic and the Poincaré sections are topologically equivalent. Figures $5 b, 5 d$, and $5 e$ show the stroboscopic sections for the three modes. These are obtained by collecting the points in the phase space at times $t_{0}, t_{0}+\tau_{\mathrm{RF}}, t_{0}+2 \tau_{\mathrm{RF}} \ldots$, where $\tau_{\mathrm{RF}}$ is the excitation period. Figures $5 \mathrm{~d}$ and 5 e show sets consisting of a single point, being a further evidence of periodicity of the respective oscillations. Figure $5 b$, however, shows a complexlooking set of points shaped like a horseshoe and this is an additional argument for the existence of a chaotic attractor in the system.

In addition to different electrical properties, the three modes have very different visual appearance. Using an Andor iStar iCCD imaging system that is synchronized to the applied voltage waveform, it is possible to take nanosecond exposure images of the plasma plume throughout each cycle of the applied voltage. Plasma imaging in the chaotic mode was found unrealistic because its temporal variation does not support image accumulation and single-shot 
images were too faint (data not shown). The space-integrated optical signatures of the two periodic modes (obtained via the detection optical fiber) are shown in figure 6, which plots the wavelength-integrated optical emission signal $(350-800 \mathrm{~nm})$ and the discharge current as a function of time. In the bullet mode, the light emission appears as a sharp burst during each half-cycle of the applied voltage and the peak intensity is observed slightly after the peak of the current pulse. Temporal pattern of the light emission appears strongly modulated at the excitation frequency, and agrees with the presence of a plasma bullet passing the detection optical fiber. It is evident in figure 6 that the emission patterns from positive and negative half cycles are different. In the positive half cycles, the optical emission appears as a highly intense yet narrow burst whereas the emission in the negative half-cycles is less intense but broader in duration. Therefore the temporal pattern of light emission in the bullet mode is more closely related to the discharge current than the applied voltage that has the same amplitude in the positive and negative half cycles. In the continuous mode on the other hand, the emission within the discharge space never drops to zero, suggesting the continuous nature in this mode of operation. This is in stark contrast to that observed in the bullet mode. Emission is low at the voltage zero crossing points and high when the voltage reaches a maximum. The light emission is modulated at the excitation frequency, however the variation of its emission amplitude from one cycle to the next does not appear to follow closely that of the discharge current nor the applied voltage in figure $2 c$. These observations suggest that optical emission signal tracks electrical signals (i.e. either voltage or current) differently in the bullet mode and the continuous mode.

Figure 7 shows a sequence of 10ns exposure images taken throughout the positive and negative half cycles of the applied voltage whilst the plasma jet was operating in the bullet mode. Images $a$ to $d$ were taken during the negative half cycle, consequently the downstream ground electrode (on the right hand side of the right graph in figure 7) can be considered as the instantaneous anode and the powered electrode wrapped around the dielectric tube as the instantaneous cathode (on the left hand side). As the current pulse increases in magnitude a 
faint bullet-like object can be observed leaving the grounded anode and traveling toward the cathode inside the quartz tube, such observations have not been reported previously for atmospheric plasma jets. Images $e$ to $h$ were taken over a positive half cycle, in this case the powered electrode was the instantaneous anode and the grounded electrode the instantaneous cathode. Several previous studies demonstrated similar images, in this situation the plasma bullet appears to be ejected from the quartz tube and travels at high velocity (up to $10^{6} \mathrm{~m} / \mathrm{s}$ ) towards the ground electrode (i.e. the instantaneous cathode) [17][19][41][42]. As all the images are normalized, it is possible to determine from figure $6 \mathrm{~b}$ that the intensity of the plasma bullet during the negative half cycle is considerably less than that in the positive half cycle. This agrees well with the data presented in figure 6 . Also worth noting is the slight difference in structure, the bullet no longer appears to have an intense centre but appears as an elongated region of moderate intensity plasma.

It should be mentioned that plasma bullets tend to form in the positive half-cycle and in the case of figure 7 the discharge in the negative half cycle does not have the appearance of a plasma bullet. However sometimes bullet-like behaviors were also observed in the negative half-cycles (data now shown). In general, there is at least one bullet every cycle of the applied voltage in the bullet mode. Also it should be mentioned that plasma bullets are cathodedirected and in this regard they are very similar to streamers. However plasma bullets appear in a well defined column without branching and their characteristics are similar to that of glow discharges. Therefore there may be some differences, yet to be fully understood, between plasma bullets and streamers.

One possible theory attributed to the plasma bullet phenomenon was the photon-ionization theory of Dawson [19][61]. Dawson's original work [61], concerning the generation of cathode-directed streamers in atmospheric pressure air, demonstrated that the streamer tip was able to propagate in regions of low electric field at very high velocity due to a photoionization effect. It was postulated that the streamer tip consists of numerous positive ions, photons emitted from the streamer tip create photoelectrons at a small distance in front of the 
tip. The high electric field between the photoelectron and positive streamer tip results in a rapid acceleration of the electron thus producing an avalanche. Provided that the avalanche produces a sufficient number of electrons, the streamer tip is neutralized completely resulting in the creation of a new positive region a small distance ahead of the original streamer tip and a quasi neutral tail. This propagation mechanism is graphically illustrated in figure 8 , and can be used to explain plasma bullet propagation of several centimeters with velocities up to $10^{6}$ $\mathrm{m} / \mathrm{s}$ [41]-[43]. Of importance here is that Dawson's theory assumes complete neutralization of positive ions by electrons produced by photon ionization. By means of a repeated process of photon-ionization and neutralization, the plasma head collapses and then regenerates itself at a short distance in front of its original location. This repetition of generation, collapse and regeneration occurs very quickly, and so on a timescale too long to resolve the process (often longer than $10 \mathrm{~ns}$ ) the plasma would appear as an illuminant and spatially confined object moving continuously to the cathode. Similarly, the plasma tail follows the plasma head closely behind since charges in the wake of the plasma head are completely neutralized [61]. Therefore complete neutralization is critical in supporting a dynamic optical pattern in which the plasma head moves with a tracking plasma tail. Dawson's original work was conducted in the absence of a dielectric barrier, this is in contrast to many of the recent studies of atmospheric plasma jets. It is likely that the presence of dielectric surfaces, which are capable of trapping electrical charges, may assist the accumulation of positive charge in the streamer/bullet tip.

While Dawson's theorem is highly plausible for explanation of plasma bullets in lowtemperature atmospheric discharge jets [19][41]-[44], no direct evidence has so far been reported of the sequentially occurring pattern of generation, collapse and regeneration of the plasma head in APGD jets. The streamer propagation mechanism postulated by Dawson's theory therefore remains speculative for APGD jets. To this end, we took single-shot images of the plasma plume in the bullet mode with a nominal exposure time of 2 ns and results are shown in figure 9. An alternating pattern of bright and pale plasma head images is evident, 
indicating a periodic burst of photon emission repeated on a time scale of approximately 2 ns or below. Through photon ionization, this periodic burst of photon emission is likely to result in a periodic generation of electrons in front of the plasma head thus creating an impression of a continuously moving plasma head on a timescale of $10 \mathrm{~ns}$ or longer as suggested in figure 7. Yet figure 9 suggests that the evolution of the plasma head is not continuous but involves a repetition of generation, collapse, and re-generation at locations progressively extending towards the instantaneous cathode. This evidence has not been reported before and offers a direct evidence of plasma bullet dynamics as postulated in Dawson's theory. It should be remarked here that the iCCD manufacturer's recommended minimum exposure time is 5 ns. A lower minimum of 2 ns can be set manually for the iCCD camera, although the precision of the 2 ns exposure time is not guaranteed. To avoid any artifacts in the 2 ns images of figure 9, we repeated the imaging process at different instants of exposure and under different plasma conditions in the bullet mode. The bright and pale pattern of figure 9 was always reproducible and should be valid. It should be mentioned however that the timescale of 2 ns in figure 9 should not be used for estimates of the timescale of plasma head generation and regeneration, as the latter should be resolved at a timescale much lower than 2 ns.

To see plasma appearance in the continuous mode, figure 10 shows a sequence of $10 \mathrm{~ns}$ exposure images taken with $V_{p}=3.9 \mathrm{kV}$. The gain of the iCCD camera was reduced so that the jet structure in the continuous mode could be compared to that in the bullet mode of figure 7 , consequently it is not possible to directly compare emission intensities here with those in figure 7. Images $a$ to $d$ were taken during the negative half cycle and images $e$ to $h$ were taken in the positive half cycle. Clearly there is no immediately apparent plasma bullet in either case; the discharge can be seen to reach from one electrode to the other throughout one entire cycle of the applied voltage. To confirm the continuous nature of the plasma plume, the iCCD exposure time was reduced to its minimum of $2 n s$. In this case, the plume still appeared continuous and this is consistent with the current measurement of figure $2 c$ and with the wavelengthintegrated optical signal of figure 6 . It is therefore reasonable to conclude that the plasma jet is 
operating in a continuous mode. The emission intensity of the discharge is time-modulated at the excitation frequency, however even at the zero crossing points of the current and voltage waveforms the iCCD camera picks up strong optical emission. As the duration of each current pulse is almost equal to that of the voltage half cycle, it is likely that the densities of energetic species in the electrode gap, especially the $2^{\text {nd }}$ positive system of nitrogen and helium metastables, remain very high throughout the entire excitation cycle. It is known that in nitrogen mixtures with noble gases collisions between $\mathrm{N}_{2}$ metastables are able to efficiently produce electrons by the Penning effect [62] (see more detailed discussion in the next section). This is supported by the rapid increase in dissipated power in the continuous mode (see figure 3). As the electrons remain in the gap, gas conductivity increases meaning that a slight increase in the applied voltage can cause a large growth in the discharge current. This phenomenon continues until arcing. Previous studies of a similar plasma jet excited from $10 \mathrm{kHz}$ to $1 \mathrm{MHz}$ showed a similar trend with increasing frequency [42]. As the frequency was increased, each discharge event became temporally closer resulting in a continuous presence of excited species in the electrode gap to support continuous electron production [42]. It was shown at high excitation frequencies the plasma plume becomes a continuous discharge [42], similar to that reported here.

The absence of plasma bullets in the continuous mode does not necessarily mean that the photon-ionization mechanism of Dawson's theory is no longer valid. As considerable helium metastables and excited nitrogen molecules exist in the electrode gap over the entire duration of an excitation cycle, electrons are produced efficiently through step-wise ionization, superelastic ionization, and metastable pooling of helium metastables as well as through Penning ionizations involving excited nitrogen molecules (see discussion in the next section). These channels of electron generation become much more effective in the continuous mode than in the bullet mode, and will significantly affect the spatial distribution of space charges to an extent that overwhelms the contribution of photon ionization. This is likely because electron generation by neutral helium and nitrogen species is relatively independent of the spatial 
location in the electrode gap and likely to occur anywhere in the electrode gap. If this is the case, then they would mask the effects of photon ionization postulated in Dawson's theory and mask the presence of a plasma bullet even if it was produced. It is clear that the continuous mode has a multiplicity of competing channels of electron generation, more complex than the bullet mode where the role of photoelectrons may be dominant. New experiment designs will therefore be needed in order to unravel the roles of different electron generation mechanisms in the continuous mode.

Images in figure 11 suggest that the plasma plume has a distinctive structure in the continuous mode. During a negative half cycle, the discharge reaches from the instantaneous anode (the downstream ground electrode) towards the instantaneous cathode (the wrapped electrode) and it appears to reduce in intensity. In the positive half cycle when the grounded electrode is the cathode, a very intense discharge is apparent near the surface of the ground electrode, then a small dark region is observed followed by a less intense region stretching toward the anode. Figure 11 provides a close-up image of the plasma near the grounded electrode taken at the current maximum during each half cycle. Figure 11a was taken during the positive half cycle when the ground electrode was the cathode, the pattern of light and dark regions resembles that of an elongated dc glow discharge structure, a highly intense negative glow region is followed by a dark region preceding a large but low intensity bulk region [63]. Such patterns have been reported previously in studies involving $\mathrm{kHz}$ excited parallel-plate atmospheric discharges [62]. In the negative half-cycle, the ground electrode becomes the anode and figure 11b shows that the structure differs considerably from that observed in the positive half cycle. No intense negative glow region or obvious dark space is observed, the lack of structural features at the anode is again typical of a glow discharge. Both images suggest that the continuous mode is very much like a typical glow discharge. 


\section{Emission Spectra Characteristics:}

Optical emission spectroscopy is highly convenient for determining several characteristics of the plasma in a non-invasive manner [64]. In all cases reported here, the fiber optic probe was placed $1 \mathrm{~mm}$ below the exit of the quartz tube where the helium flow from the jet structure was mixed with the ambient air thus introducing significant nitrogen and oxygen impurities. Spectral data was intensity-calibrated using a NIST traceable white light source to ensure emission intensities remain unaffected by the spectral response of the system. Figure $12 \mathrm{a}$ and $12 \mathrm{~b}$ show typical emission spectra obtained whilst the plasma jet is in the bullet mode and the continuous mode, respectively, with key emission lines labeled. When the ionized helium gas interacts with the ambient air, highly energetic helium metastables are able to cause ionization of nitrogen [65]. It is of no surprise that nitrogen species are dominant in such a gas mixture; the helium discharge within the quartz tube produces a large flux of long lifetime helium metastables. However, due to the rapid Penning reaction [65]:

$$
\begin{aligned}
& \mathrm{He}^{*}+\mathrm{N}_{2} \rightarrow \mathrm{rN}_{2}^{+}\left(\mathrm{B}^{2} \Sigma_{\mathrm{u}}^{+}\right)+(1-\mathrm{r}) \mathrm{N}_{2}^{+}\left(\mathrm{X}^{2} \Sigma_{\mathrm{g}}^{+}\right)+\mathrm{He}+e \quad k_{1}=7.6 \times 10^{-11} \mathrm{~cm}^{3} \mathrm{~s}^{-1} \\
& \mathrm{He}_{2}{ }^{*}+\mathrm{N}_{2} \rightarrow \mathrm{rN}_{2}^{+}\left(\mathrm{B}^{2} \Sigma_{\mathrm{u}}^{+}\right)+(1-\mathrm{r}) \mathrm{N}_{2}^{+}\left(\mathrm{X}^{2} \Sigma_{\mathrm{g}}^{+}\right)+2 \mathrm{He}+e \quad k_{1}=7.0 \times 10^{-11} \mathrm{~cm}^{3} \mathrm{~s}^{-1}
\end{aligned}
$$

the effective lifetimes of helium metastables become short, resulting in a rapid reduction of helium metastable density when the helium discharge meets the ambient air. Additionally, the abundance of excited nitrogen species grows rapidly in the continuous mode resulting in strong nitrogen emission as indicated in figure 12. Emission intensities of the excited $\mathrm{N}_{2}$ at $357 \mathrm{~nm}$ and $\mathrm{N}_{2}{ }^{+}\left(\mathrm{B} \Sigma_{\mathrm{u}}-\mathrm{X} \Sigma_{\mathrm{g}}\right)$ ions at $391 \mathrm{~nm}$ are shown as functions of dissipated power in figure 12c, where the $\mathrm{N}_{2}$ emission intensity is seen to increase in an approximately linear fashion with the dissipated power. The $\mathrm{N}_{2}{ }^{+}$emission intensity increases rapidly in the chaotic mode then starts to level off in the bullet mode before undergoing a gradual decay well into the continuous mode. From an application stand point, the rapid growth in excited nitrogen species is beneficial, a very stable but inert helium plasma is produced to which molecules of a reactive gas are added downstream, thus resulting in a chemically active plasma without the need to 
directly ionize highly reactive and unstable molecular gases. Many applications rely heavily upon excited oxygen and nitrogen species in the discharge to influence reaction chemistry [66][67]. For example, in the field of biological decontamination it is known that atomic oxygen species play an important role in the bacterial and protein inactivation mechanism [67][68]; however, discharges employing reactive gases often prove too hot to be useful, in such a situation a reactive yet low temperature helium plasma jet is highly desirable.

In order to further our understanding of how the discharge behaves in each mode of operation, it is useful to consider the time evolution of key chemical species of the helium plasma jet. Figure 13 provides wavelength filtered nanosecond images of the bullet and the continuous modes. Using band pass filters centered on 390nm and 710nm (Thorlabs FWHM $10 \mathrm{~nm})$, it was possible to track the $\mathrm{N}_{2}{ }^{+}$emission at $391 \mathrm{~nm}\left(18.77 \mathrm{eV}\right.$ from ground state $\mathrm{N}_{2}$ ) and the He emission at $706 \mathrm{~nm}\left(3 \mathrm{~s}^{3} \mathrm{~S}\right.$ state, threshold energy of $\left.22.7 \mathrm{eV}\right)$. Figure 13 a shows the intensity profile for each emission line whilst operating in the bullet mode and the downstream electrode being the instantaneous cathode. The images were taken shortly after the peak current and the plasma bullet was just free of the quartz tube. Figure 13a indicates that helium emission leads $\mathrm{N}_{2}^{+}$emission in the direction towards the downstream electrode (instantaneous cathode). It is known that the helium 706nm line is indicator of energetic electrons [65], and therefore figure $13 \mathrm{a}$ implies that the front of the plasma bullet is largely made of high energy electrons with a cloud of positive nitrogen ions located at a short distance behind the electron region. Nitrogen ions behind the electron cloud, shown in the wavelength-filtered image in figure 13a, are likely to be produced from Penning ionization of nitrogen from helium metastables (see eq. $(1,2)$ ). It should be noted that other positive ions are also produced in atmospheric helium plasmas in contact with nitrogen, for example $\mathrm{He}^{+}, \mathrm{He}_{2}{ }^{+}, \mathrm{N}^{+}, \mathrm{N}_{3}{ }^{+}$and $\mathrm{N}_{4}{ }^{+}$. Several numerical simulation of low-current regimes of RF atmospheric $\mathrm{He}-\mathrm{N}_{2}$ discharges considered $\mathrm{He}^{+}, \mathrm{He}_{2}{ }^{+}, \mathrm{N}^{+}, \mathrm{N}_{2}^{+}$and found that dimmer ions are more numerous [50][69]. In particular, $\mathrm{N}_{2}{ }^{+}$and $\mathrm{He}_{2}{ }^{+}$concentrations were found to be in the order of $10^{11} \mathrm{~cm}^{-3}$ and $10^{10} \mathrm{~cm}^{-3}$ [50], respectively, in the low-current regime of the $\alpha$ mode which could be considered as 
equivalent to the case of atmospheric DBDs in the same $\mathrm{He}-\mathrm{N}_{2}$ mixture. The important role of $\mathrm{N}_{2}{ }^{+}$was also strongly supported in some numerical studies of atmospheric DBDs [70]-[72], though they did not consider contributions of $\mathrm{N}_{3}{ }^{+}$and $\mathrm{N}_{4}{ }^{+}$. A more recent study of atmospheric DBD in He- $\mathrm{N}_{2}$ mixture suggested that $\mathrm{N}^{+}$and $\mathrm{N}_{3}{ }^{+}$ions play a minor role in comparison to $\mathrm{N}_{2}{ }^{+}$ and $\mathrm{N}_{4}{ }^{+}$ions [73]. This is because $\mathrm{N}^{+}$is rapidly converted to $\mathrm{N}_{2}{ }^{+}$through $\mathrm{N}^{+}+\mathrm{N}+\mathrm{He} \rightarrow \mathrm{N}_{2}{ }^{+}+\mathrm{He}$ and $\mathrm{N}_{3}{ }^{+}$degrades much faster than $\mathrm{N}_{4}{ }^{+}$, both via $\mathrm{N}$ [74]. Therefore the dominant ions in atmospheric He- $\mathrm{N}_{2}$ plasmas are likely to be $\mathrm{He}_{2}{ }^{+}, \mathrm{N}_{2}{ }^{+}$and $\mathrm{N}_{4}{ }^{+}$. While there is at present very little direct measurement of their concentrations, numerical simulation suggests that $\mathrm{N}_{2}^{+}$are the most numerous when $\mathrm{N}_{2}>1 \mathrm{ppm}$ [72]. Given that the ambient nitrogen influx and the nitrogen trace amount in the helium carrier gas are certain to amount to more than $1 \mathrm{ppm}$ in the plasma jet, the dominant ions in the plasma jet are likely to be $\mathrm{N}_{2}{ }^{+}$ions. Though oxygen ions need to be evaluated also, recent direct measurement of a similar atmospheric plasma jet offers a strong support for the dominant role of $\mathrm{N}_{2}^{+}[75]$. Therefore the wavelength-filtered image of $\mathrm{N}_{2}{ }^{+}$at $391 \mathrm{~nm}$ of figure 13a captures the role of positive ions in the formation of plasma bullets. It offers a direct evidence to support Dawson's theory of photon-ionization (see figure 8). A final observation of figure 13 is that light emission extends all the way back to the anode, suggesting the tail of the plasma bullet forms some kind of weak link to the electrode, at least up to the moment the photograph was taken. This is consistent with figure 7 and with our previously reported observation [42].

Figure 13b shows wavelength filtered 10 nanosecond images taken in the continuous mode of operation obtained shortly after the peak positive current pulse. In this situation, the helium emission still leads the nitrogen emission in the direction of the plasma plume extension towards the downstream electrode. The intensity profiles indicate a very intense region above the cathode followed by a rapid reduction in the intensities of $\mathrm{He}$ and $\mathrm{N}_{2}{ }^{+}$. Notably, the total emission does not decline as quickly suggesting that other species such as nitrogen molecules and oxygen atoms may play a significant role. This is in agreement with the emission spectrum presented in figure 12 which shows a dominance of nitrogen and oxygen species. The 
wavelength filtered images indicate that highly energetic species are confined to a small region around the cathode, consistent with the assumption that this is a negative glow region where the electric field is high. As the time averaged spectra in figure 12 show the nitrogen second positive emission to be significant, it is possible that these species are capable of producing a significant amount of seed electrons for subsequent discharge events. Indeed, Gherardi and colleagues investigated the transition between glow and filamentary operating modes in an atmospheric pressure nitrogen dielectric barrier discharge and determined that the influence of seed electrons produced by the Penning effect between two nitrogen molecules is crucial in maintaining a stable glow discharge [62]:

$$
\begin{array}{ll}
\mathrm{N}_{2}\left(\mathrm{a}^{\prime 1} \Sigma_{\mathrm{u}}^{-}\right)+\mathrm{N}_{2}\left(\mathrm{~A}^{3} \Sigma_{\mathrm{u}}^{+}\right) \rightarrow \mathrm{N}_{4}^{+}+\mathrm{e} & k_{2}=5 \times 10^{-11} \mathrm{~cm}^{3} \mathrm{~s}^{-1} \\
\mathrm{~N}_{2}\left(\mathrm{a}^{\prime 1} \Sigma_{\mathrm{u}}^{-}\right)+\mathrm{N}_{2}\left(\mathrm{~A}^{\prime 1} \Sigma_{\mathrm{u}}^{-}\right) \rightarrow \mathrm{N}_{4}^{+}+\mathrm{e} & k_{3}=2 \times 10^{-11} \mathrm{~cm}^{3} \mathrm{~s}^{-1}
\end{array}
$$

$\mathrm{N}_{4}{ }^{+}$ion production was found to increase monotonically with $\mathrm{N}_{2}$ admixture in helium and hence electron production via the two $\mathrm{N}_{2}\left(\mathrm{a}^{\prime} \Sigma_{\mathrm{u}}{ }^{-}\right)$routes is expected to increase also [73]. The increase in $\mathrm{N}_{4}{ }^{+}$concentration is about a factor of 10 when $\mathrm{N}_{2}$ admixture increases from 1ppm to $5 \mathrm{ppm}$ [73]. Other electron generation reactions include [69][76]:

$$
\begin{array}{ll}
\mathrm{He}^{*}+\mathrm{He}^{*} \rightarrow \mathrm{He}^{+}+\mathrm{He}+\mathrm{e} & k_{4}=2.7 \times 10^{-10} \mathrm{~cm}^{3} / \mathrm{s} \\
\mathrm{He}_{2}{ }^{*}+\mathrm{N}_{2} \rightarrow \mathrm{N}_{2}{ }^{+}+2 \mathrm{He}+\mathrm{e} & k_{6}=7 \times 10^{-11} \mathrm{~cm}^{3} / \mathrm{s} \\
\mathrm{He}_{2}{ }^{*}+\mathrm{He}_{2}{ }^{*} \rightarrow \mathrm{He}^{+}+2 \mathrm{He}+\mathrm{e} & \mathrm{k}=1.5 \times 10^{-9} \mathrm{~cm} 3 / \mathrm{s}
\end{array}
$$

For RF atmospheric He- $\mathrm{N}_{2}$ discharges, $\mathrm{He}_{2}{ }^{*}$ concentration is usually two orders of magnitude above He* concentration over a wide range of discharge current [50]. Given that the reaction coefficients in (4) are within one order of magnitude and that concentration of $\mathrm{N}_{2}$ admixture is usually higher than $\mathrm{He}^{*}$ and $\mathrm{He}_{2}{ }^{*}$ concentration (typically $\sim 10^{12} \mathrm{~cm}^{-3}$ ), Penning ionization of (3b) is expected to make a major contribution to electron production. This is likely to hold true for atmospheric He- $\mathrm{N}_{2}$ DBDs also. As the discharge current increases from the $\alpha$ mode to the $\gamma$ mode in RF discharges, $\mathrm{He}_{2}{ }^{*}$ concentration can increase by a factor of 10 , consequently reaction $3 \mathrm{c}$ may begin to play an important role [50]. The above discussions highlight that as increasing current evolves the discharge into the continuous mode many electron production 
channels become significant. Their contributions are likely to become comparable or exceed the contribution of photon ionization, thus masking the latter in the optical appearance of the plasma plume. Therefore even if the photon ionization mechanism of Dawson's theory still support plasma bullet formation in the continuous mode, the bullet appearance would have been masked by other electron production processes.

As the plasma evolves from the chaotic mode, through the bullet mode, to the continuous mode, its electrical and optical properties (see figure $2-4,6$ ), its phase portrait and stroboscopic sections (figure 5), its structures (see figure 7, $9-11$ ), and its formation mechanisms (see figure 12 - 13) undergo abrupt and distinct changes. Reaction chemistry driven by these distinct changes is seen however to vary in a more gradual fashion as shown in figure 12. In principle, more abundant reaction species are produced at large dissipated power and inevitably the latter raises the gas temperature that could become unacceptable for some applications. To provide an indication of gas temperature, we consider optical emission spectrum of nitrogen bands as they are well established to determine rotational and vibrational temperatures of nitrogen containing discharges [77][78]. The highly collisional nature of atmospheric pressure plasmas means that it is reasonable to assume that the rotational temperature of the gas is in equilibrium with its translational temperature; as such the rotational temperature is a useful indicator of gas temperature [79]. In order to determine rotational and vibrational temperatures, the experimental spectra of the nitrogen emission band $\left(C^{3} \Pi_{u}-B^{3} \Pi_{g}\right)$ around $380 n m$ were compared to simulation data. All simulation data were produced using Specair [77]. By defining the input parameters of $T_{\text {rot }}$ and $T_{\text {vib, }}$ and the spectrometer slit function, it was possible to closely match the experimental results. Figure $14 a$ shows experimental data obtained at a dissipated power of $3 \mathrm{~W}$, the simulation data are overlaid on the experimental data and indicate a best-fit rotational temperature of $410 \mathrm{~K}$ and a vibrational temperature of $3,500 \mathrm{~K}$. Figure $14 \mathrm{~b}$ shows the trend in rotational temperature with increasing dissipated power. At the point of breakdown, the rotational temperature is found to be $310 \mathrm{~K} \pm 20 \mathrm{~K}$, such temperatures are commonly observed in plasma jets of this type and are 
well suited for low temperature materials processing. As input power is increased from $0.4 \mathrm{~W}$ to $0.75 \mathrm{~W}$, the rotational temperature rises from $310 \mathrm{~K}$ to $340 \mathrm{~K}$. As the discharge is in the chaotic mode, any increase in power results in an increase in the number of discharge events, hence it is expected that the gas temperature would increase during this mode. From $0.75 \mathrm{~W}$ to $1.3 \mathrm{~W}$, the temperature increases slightly from $340 \mathrm{~K}$ to $350 \mathrm{~K}$, suggesting that the gas temperature in the bullet mode of operation varies little with input power. Finally, as the discharge transitions from the bullet mode to the continuous mode the gradient of gas temperature is seen to increase indicating enhanced gas heating. This notable shift in gas temperature between modes of operation is likely a result of the difference in duration over which the gas discharge is sustained. In the bullet mode, the discharge current persists for a fraction of the excitation cycle (see figure $2 \mathrm{~b}$ ) and so the dissipated power, as reflected in the product of the current density and electric field $J \cdot E$, is significant only for a short period of time, thus limiting possible gas heating. In the continuous mode the background gas is ionized throughout the entire applied voltage cycle meaning the background gas is heated all the time. It is worth pointing out that temperatures above $350 \mathrm{~K}$ may become undesirable for treatment of thermally liable substrates.

\section{Concluding Remarks:}

Based on a detailed electrical, fast imaging, and spectroscopic characterization of a linear-field atmospheric plasma jet, this study has provided coherent evidence of the existence of three operating modes, namely the chaotic mode, the bullet-mode and the continuous mode from plasma ignition to arcing. With increasing dissipated power after gas breakdown, the plasma jet has been shown to evolve through the three modes of distinct differences and abrupt mode transition. The chaotic mode occurs immediately after the gas breakdown point, and its discharge current has a chaotic temporal character with some of its negative half-cycles free of any discharge event. This has been shown to be related to a weak gas discharge and its 
inadequate supply of seed electrons, causing an inability to support a dynamic balance between the applied voltage and the memory voltage that is essential for stable and periodic plasma in a DBD. With increasing input power to enhance the level of gas discharge, plasma dynamics become periodic and the plasma jet enters its bullet mode where there is one strong plasma bullet every positive half cycle. Dawson's photon-ionization theory has been studied and supported with time- and wavelength-resolved 2D plasma images that provide evidence of electrons being produced in front of the plasma bullet tip with the bullet composed of mainly $\mathrm{N}_{2}{ }^{+}$ions. Possible contributions of other positive ions have also been discussed in comparison with the role of $\mathrm{N}_{2}^{+}$ions. The evolution of the plasma bullet has been shown to involve a repetition of generation, collapse and regeneration of the plasma head at locations progressively extending towards the instantaneous cathode. A further increase in input power has been demonstrated to eventually evolve the plasma jet into its continuous mode, in which both the discharge current and the wavelength-integrated optical emission remain high from one half-cycle to the next. Electron generation by helium metastables and excited nitrogen molecules has been found to be significant and possibly independent of the spatial location between the electrode gap. These may mask the signature of photon ionization and contribute to plasma bullets not being observed in the continuous mode. Overall, this work provides a detailed study of characteristics and possible mechanisms of all three modes of operation in an atmospheric plasma jet. It is expected that similar modes and mode transition are also present in cross-field plasma jets and indeed other types of plasma jets, with one exception of RF or other high-frequency devices in which the bullet mode may be absent [42].

This study has brought to light several interesting observations, for example the cathodedirected plasma moving towards the quartz tube, the structural difference of the plasma root on the grounded electrode for the two possible voltage polarities, and the different temporal relations of optical emission to the applied voltage in the two periodic modes. Some of these observations highlight the need for further fundamental studies, and indeed more direct measurements are necessary to fully characterize the three modes. Nevertheless, observations 
presented here and their discussions do form a coherent understanding of how plasma dynamics and reaction chemistry evolve in an atmospheric plasma jet from its gas breakdown to the formation of plasma bullets and glow discharge and to its arcing. This offers a previously unavailable insight into plasma jet formation mechanisms and a potential of matching plasma jet characteristics to specific needs of an intended application. Both are important goals in the current research of cold atmospheric plasma jets. 


\section{References:}

[1] Becker K H, Schoenbach K H, Eden JG 2006 J Phys D Appl Phys 39 R55.

[2] Tachibana K 2006 IEEJ Trans 1145.

[3] Tendero C, Tixier C, Tristant P, Desmaison J and Leprince P 2006 Spectrochimica Acta B 612.

[4] Iza F, Kim G J, Lee S M, Lee J K, Walsh J W, Zhang Y T and Kong M G 2008 Plasma Process Polym 5322.

[5] Noeske M, Degenhardt J, Strudthoff S, and Lommatzsch U 2004 Int J Adhes Adhes 24, 171.

[6] Girard-Lauriault PL, Mwale F, lordanova M, Demers C, Desjardins P and Wertheimer MR 2005 Plasma Process Polym 2263.

[7] Laroussi M 2002 IEEE Trans Plasma Sci 30, 1409.

[8] Deng X T, Shi J J and Kong M G 2007 J Appl Phys 101074701.

[9] Stoffels E 2002 High Temp Mat Processes 6191.

[10] Fridman G, Friedman G, Gutsol A, Shekhter AB, Vasilets VN and Fridman A 2008 Plasma Processes and Polymers 503.

[11] Chen CK, Perry WL, Xu H, Jiang Y and Phillips J 2003 Carbon 412555.

[12] Richmonds C and Sankaran RM 2008 Appl Phys Lett 93131501.

[13] Walsh J L, Shi J J and Kong M G 2006 Appl Phys Lett 88171501.

[14] Shi W, Stark RH and Schoenbach KH 1999 IEEE Trans Plasma Sci 2716.

[15] Feng H, Sun P, Chai Y, Tong G, Zhang J, Zhu W and Fang J 2009 IEEE Trans Plasma Sci 37, 121.

[16] Walsh J L and Kong M G 2007 Appl Phys Lett 91, 221502.

[17] Teschke M, Kedzierski J, Finantu-Dinu EG, Korzec D and Engemann J 2005 IEEE Trans Plasma Sci 33, 310.

[18] Yu H, Perni S, Shi JJ, Wang DZ, Kong MG and Shama G 2006 J Appl Microbio 1011323.

[19] Lu X and Laroussi M $2006 \mathrm{~J}$ Appl Phys 100, 063302.

[20] Babayan SE, Jeong JY, Schutze A, Tu VJ, Moravej M, Selwyn GS and Hicks RF 2001 Plasma Sources Sci Technol 10, 573.

[21] Bardos L, Barankova H and Berg S 1997 Surf Coat Technol 97, 723.

[22] Sakiyama Y and Graves DB 2006 J Phys D Appl Phys 393644.

[23] Kim DB, Rhee J K, Gweon B, Moon SY and Choe W 2007 Appl Phys Lett 91, 151502.

[24] Benedikt J, Focke K, Yanguas-Gil A, and von Keudell A 2008 Appl Phys Lett 92251504.

[25] Kuo SP, Koretzky E and Orlick L 1999 IEEE Trans Plasma Sci 27752.

[26] Kikuchi T, Hasegawa Y and Shirai H 2004 J Physics D Appl Phys 37, 1537.

[27] Kim J and Terashima K 2005 Appl Phys Lett 86, 191504.

[28] Czylkowski D, Jasinski M, Mizeraczyk J and Zakrzewski Z 2006 Czechoslovak J Phys 56 B684.

[29] Shimizu T, Steffes B, Pompl R, Jamitzky F, Bunk W, Ramrath K, Georgi M, Stolz W, Schmidt HU, Urayama T, Fujii S and Morfill GE 2008 Plasma Processes and Polymers 557.7

[30] Choi J, Iza F, Do H J, Lee J K and Cho MH 2009 Plasma Sources Sci Technol 18025029.

[31] Liu DW, Shi J J and Kong M G 2007 Appl Phys Lett 90, 041502.

[32] Liu DW, Iza F and Kong M G 2009 Plasma Process Polymers 6, 446.

[33] Balcon N, Aanesland A and Boswell R 2007 Plasma Sources Sci Technol 16, 217.

[34] Shi J J, Zhang J, Qiu G, Walsh J L and Kong M G 2008 Appl Phys Lett 93041502.

[35] Kong M G and Deng X T 2003 IEEE Trans Plasma Sci 317.

[36] Walsh J L and Kong M G 2006 Appl Phys Lett 89231503.

[37] Walsh J L and Kong M G 2007 Appl Phys Lett 91, 251504.

[38] Lu XP, Jiang ZH, Xiong Q, Tang ZY, Hu XY and Pan Y 2008 Appl Phys Lett 92, 151504.

[39] Boeuf J P 2003 J Physics D Appl Phys 36 R53.

[40] Adler F and Müller S $2000 \mathrm{~J}$ Phys D: Appl Phys 33, 1705.

[41] Shi J, Zhong F, Zhang J, Liu DW and Kong M G 2008 Phys Plasmas 15, 013504.

[42] Walsh J L and Kong M G 2008 IEEE Trans Plasma Sci 36, 1314.

[43] Mericam-Bourdet N, Laroussi M, Begum A and Karakas E 2009 J Physics D Appl Phys 42, 055207.

[44] Shashurin A, Shneider MN, Dogariu A, Miles RB and Keidar M 2009 Appl Phys Lett 94, 231504

[45] Walsh JW and Kong M G 2008 Appl Phys Lett 93111501

[46] Deng X T and Kong M G 2004 IEEE Trans Plasma Sci 32, 1709

[47] Abarbanel H D I 1996 Analysis of Observed Chaotic Data (New York: Springer-Verlag)

[48] Massines F, Rabehi A, Decomps P, Gadri RB, Segur P and Mayoux C 1998 J Appl Phys 83, 2950.

[49] Shi J J, Deng X T, Hall R, Punnett JD and Kong M G 2003 J Appl Phys 946303.

[50] Shi J J and Kong M G 2005 J Appl Phys 97, 023306.

[51] Ladyzhenskaya O A 1972 J Sov Math 3, 458.

[52] Ladyzhenskaya O A 1973 Sov Phys (Doklady) 17, 647. 
[53] Mallet-Paret J 1976 J Differ Equations 22, 331.

[54] Ruelle D 1982 Ann Math 115, 243.

[55] Mane R 1980 Dynamical Systems and Turbulence (Warwick) ed Rand D and Young L S (Berlin:SpringerVerlag), p 230.

[56] Packard N, Crutchfield J, Farmer D and Shaw R 1980 Phys Rev Lett 45, 712.

[57] Takens F 1981 Dynamical Systems and Turbulence ed D A Rand and Young L S Lecture Notes in Mathematics, 898 (Springer-Verlag) p 366-381

[58] Sauer T, Yorke J A, and Casdagli M 1991 J Statistical Physics 65, 579.

[59] 2003 Chaos and its Reconstruction, Ed. Gouesbet $\mathrm{G}$ et al. (Nova Science Publishers) .

[60] Fraser A and Harry L. Swinney 1986 Phys. Rev. A 33, 1134.

[61] Dawson GA and Winn WP 1965 Zeitschrift Fur Physik 183, 159.

[62] Gherardi N, Gouda G, Gat E, Ricard A and Massines F 2000 Plasma Sources Sci Technol 9, 340.

[63] Lieberman MA and Lichtenberg MA 2005 Principles of Plasma Discharges, 2nd ed._(New Jersey: John Wiley \& Sons Inc)

[64] Machala Z, Janda M, Hensel K, Jedlovsky I, Lestinska L, Foltin V, Martisovits V and Morvova M $2007 \mathrm{~J}$ Mol Spectrosc 243, 194.

[65] Bibinov NK, Fateev AA and Wiesemann K 2001 J Phys D Appl Phys 34, 1819.

[66] Jeong JY, Babayan SE, Tu VJ, Park J, Henins I, Hicks RF and Selwyn GS 1998 Plasma Sources Sci Technol 7, 282.

[67] Perni S, Shama G, Hobman J L, Lund PA, Kershaw C J, Hidalgo-Arroyo GA, Penn CW, Deng X T, Walsh J L and Kong M G 2007 Appl Phys Lett 90, 073902.

[68] Deng X T, Shi J J, Chen HL and Kong M G 2007 Appl Phys Lett 90, 013903.

[69] Yuan X and Raja LL 2003 IEEE Trans Plasma Sci 31, 495.

[70] Golubovskii YB, Maiorov VA, Behnke J and Behnke JF 2003 J Phys D Appl Phys 36, 39.

[71] Radu I, Bartnikas R and Wertheimer MR 2004 IEEE Trans Plasma Sci 31, 1363.

[72] Mangolini L, Anderson C, Heberlein J and Kortshagen U 2004 J Phys D Appl Phys 37, 1021.

[73] Martens T, Bogaerts A, Brok WJM and Dijk J 2008 Appl Phys Lett 92, 041504.

[74] Kossyi A, Kostinsky A, Matveyev A, Silakov VP 1992 Plasma Sources Sci Technol 1, 207.

[75] Urabe K, Ito Y, Tachibana K, Ganguly, BN 2008 Appl Phys Express 1, 066004.

[76] Deloche R, Monchicourt P, Cheret M, and Lambert F 1976 Phys Rev A, Gen Phys 13, 1140.

[77] Laux CO, Spence TG, Kruger CH, and Zare RN 2003 Plasma Sources Sci Technol 12, 125.

[78] Wang Q, Doll F, Donnelly VM, Economou DJ, Sadeghi N and Franz GF 2007 J Phys D Appl Phys 40, 4202.

[79] Bibinov N K, Fateev A A and Wiesemann K 2001 Plasma Sources Sci Technol 10, 579. 


\section{Figure Caption:}

Figure 1: (a) Schematic showing the structure of the jet electrode unit; and (b) an image of an atmospheric plasma jet in a $5 \mathrm{~s} / \mathrm{m}$ helium flow and at $1 \mathrm{~W}$ input power.

Figure 2: Current and voltage traces in (a) the chaotic mode, (b) the bullet mode, and (c) the continuous mode.

Figure 3: Dissipated plasma power against the RMS applied voltage, showing the operation regimes of all three distinct modes and their abrupt mode transition points.

Figure 4: Average power spectra for the chaotic, bullet and continuous modes. The excitation frequency and its harmonics are indicated in the plots.

Figure 5: $(a, c, e)$ Phase portraits and $(b, d, f)$ stroboscopic sections for the chaotic, bullet and continuous modes, respectively.

Figure 6: Wavelength-integrated $(350-800 \mathrm{~nm}$ ) emission intensity and the discharge current in (a) the bullet mode and (b) the continuous mode, each over two cycles of the applied voltage.

Figure 7: Plasma jet in the bullet mode with the current and voltage traces (left graph) and 10ns exposure images over one complete cycle of the applied voltage (right graph). In (a) of the right graph, the quartz tube is indicated with two horizontal lines on the left and the downstream electrode with a vertical line on the right.

Figure 8: Dawson's photon-ionization theory illustrated, assuming compete neutralization of positive ions by photoelectrons. Instantaneous cathode is on the right. It starts with (a) a positively changed plasma head (position \#1) emitting photons; (b) new electrons produced by photon ionization set up a strong electric field; (c) photoelectrons moving to the original plasma head and leaving behind new positive ions to form the new plasma head (position \#2); (d) ions are completely neutralized; (e) the new plasma head emits photons; (f) this creates a newer plasma head (position \#3) forming a moving plasma head.

Figure 9: Single-shot 2 ns images of a plasma bullet travelling towards the downstream ground electrode which was the instantaneous cathode.

Figure 10: Plasma jet in the continuous mode with the current and voltage traces (left graph) and 10ns exposure images over one complete cycle of the applied voltage (right graph). The quartz tube and the downstream electrode are marked the same way as in figure 7.

Figure 11: 10ns exposure images showing close-up view of the plasma jet operating in the continuous mode during (a) a positive half cycle and (b) a negative half cycle.

Figure 12: Optical emission spectrum from the atmospheric plasma jet in (a) the bullet mode; (b) the continuous mode; and (c) dissipated power dependence of emission intensities of the excited nitrogen dimmers $(357 \mathrm{~nm})$ and nitrogen dimmer ions (391 nm). The two vertical dashed lines in (c) indicate the transition points from the chaotic to the bullet modes and from the bullet to the continuous modes. 
Figure 13: Wavelength filtered intensity profiles for (a) the plasma bullet mode; and (b) the continuous mode. The instantaneous anode is the wrapped electrode and the instantaneous cathode is the downstream ground electrode, in both cases. In the bullet mode, the plasma bullet is moving from the anode to the cathode.

Figure 14: (a) Experimental and simulated nitrogen band data used to determine rotational temperature $\left(T_{\text {rot }}\right)$ and vibrational temperature $\left(T_{\text {vib }}\right)$; $(b)$ dissipated power dependence of the rotational temperature. All measured at the plasma jet contact point on the ground electrode. The two vertical lines in (b) indicate the two mode transition points. 


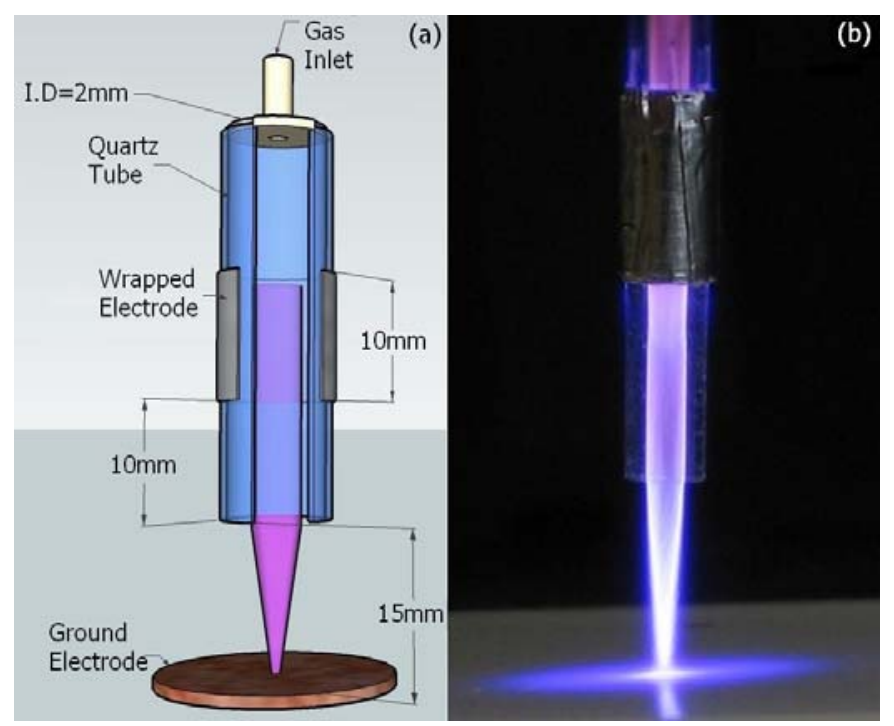

Figure 1: (a) Schematic showing the structure of the jet electrode unit; and (b) an image of an atmospheric plasma jet in a $5 \mathrm{~s} / \mathrm{m}$ helium flow and at $1 \mathrm{~W}$ input power. 


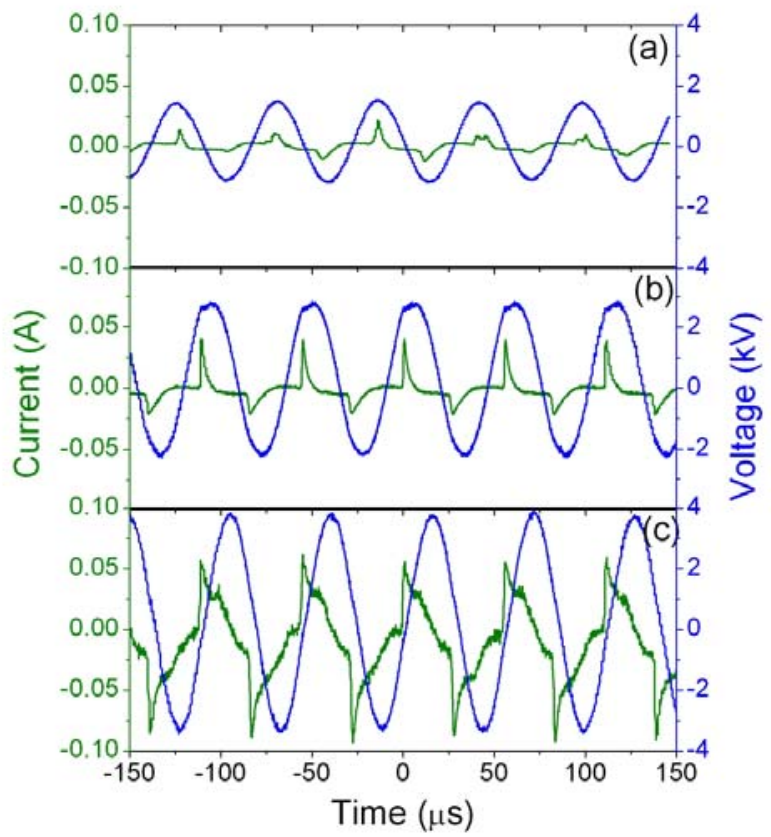

Figure 2: Current and voltage traces in (a) the chaotic mode, (b) the bullet mode, and (c) the continuous mode. 


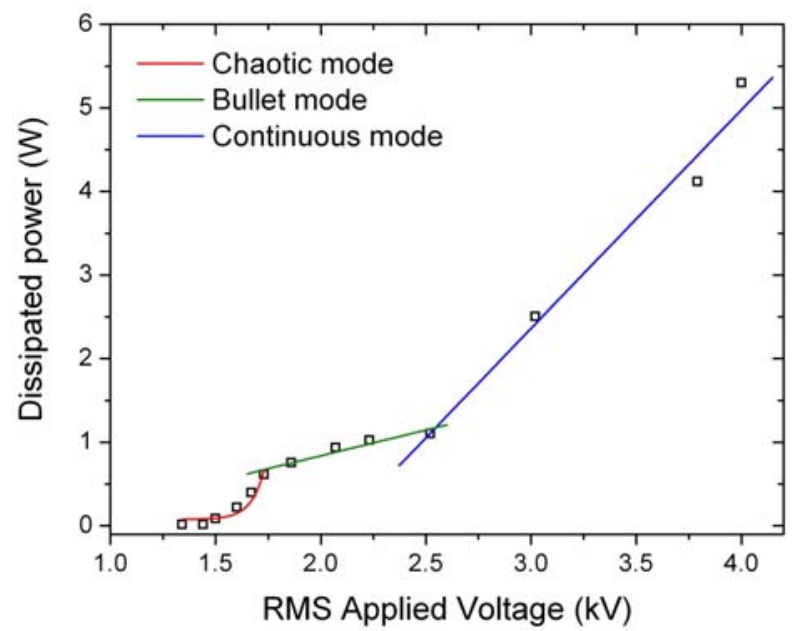

Figure 3: Dissipated plasma power against the RMS applied voltage, showing the operation regimes of all three distinct modes and their abrupt mode transition points. 


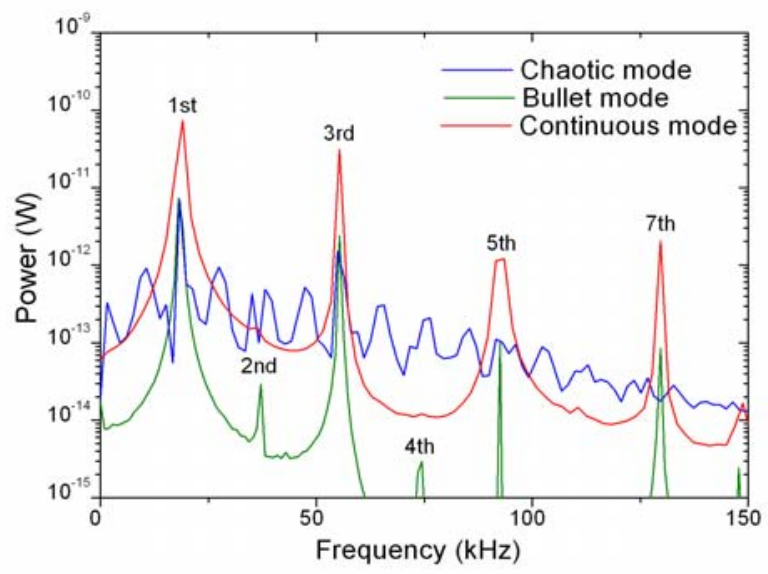

Figure 4: Average power spectra for the chaotic, bullet and continuous modes. The excitation frequency and its harmonics are indicated in the plots. 
(a)

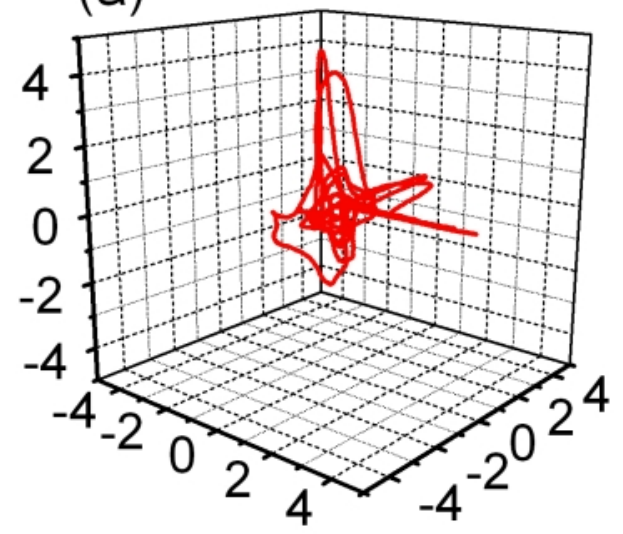

(c)

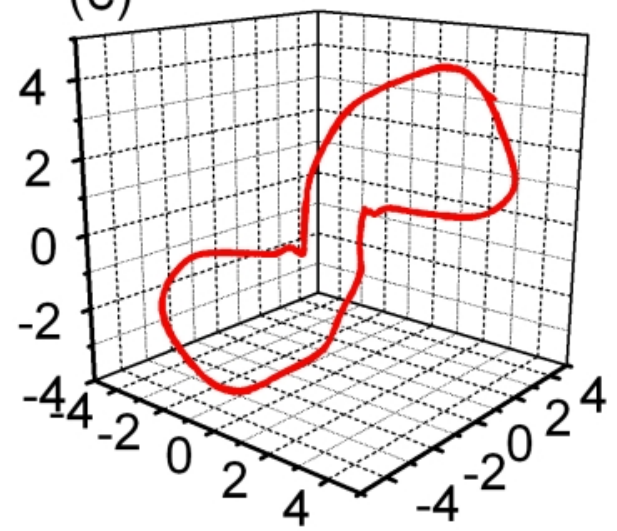

(e)

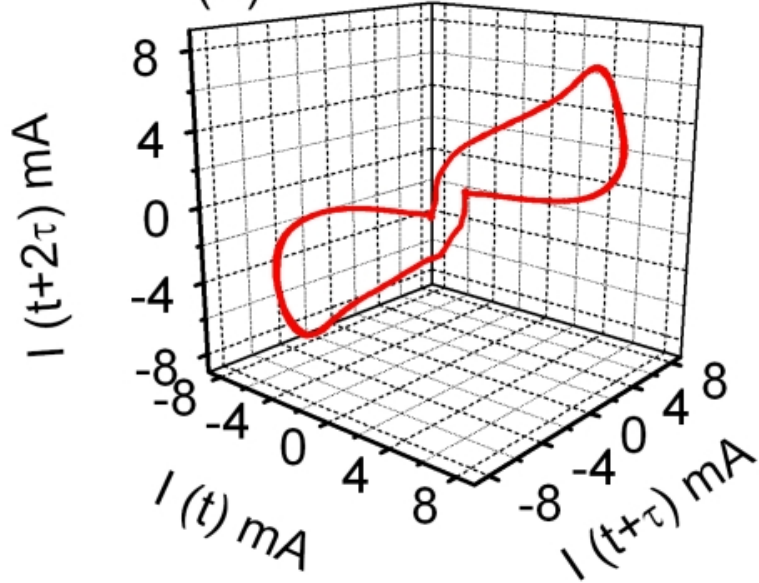

(b)

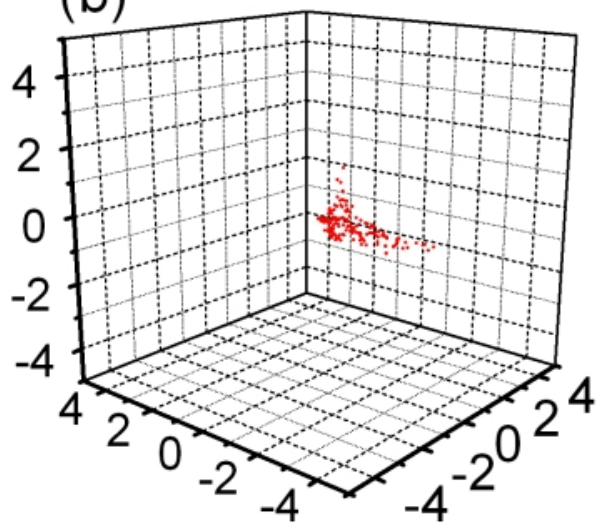

(d)

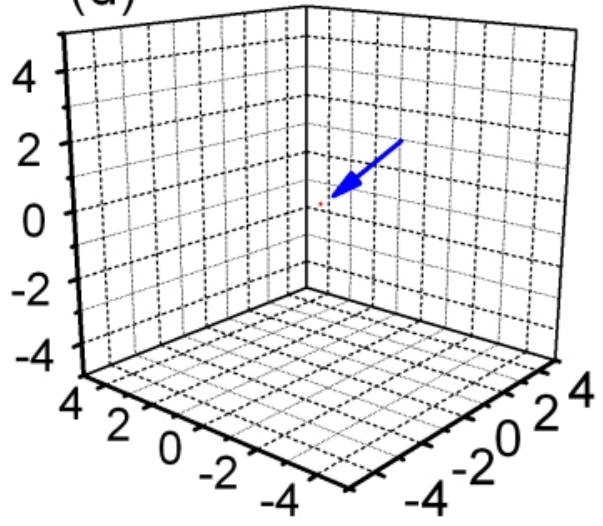

(f)

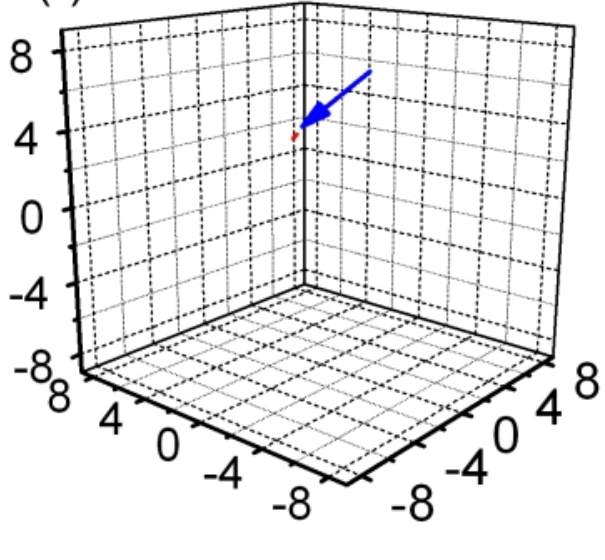

Figure 5: (a,c,e) Phase portraits and $(b, d, f)$ stroboscopic sections for the chaotic, bullet and continuous modes, respectively. 


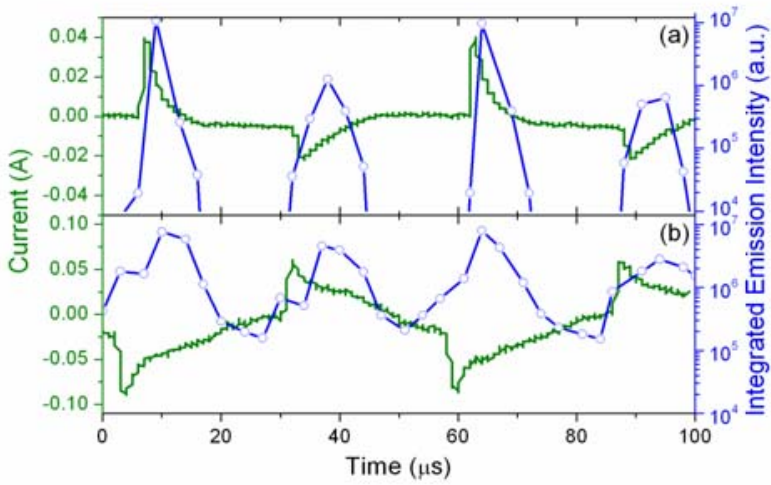

Figure 6: Wavelength-integrated $(350-800 \mathrm{~nm})$ emission intensity and the discharge current in (a) the bullet mode and (b) the continuous mode, each over two cycles of the applied voltage. 


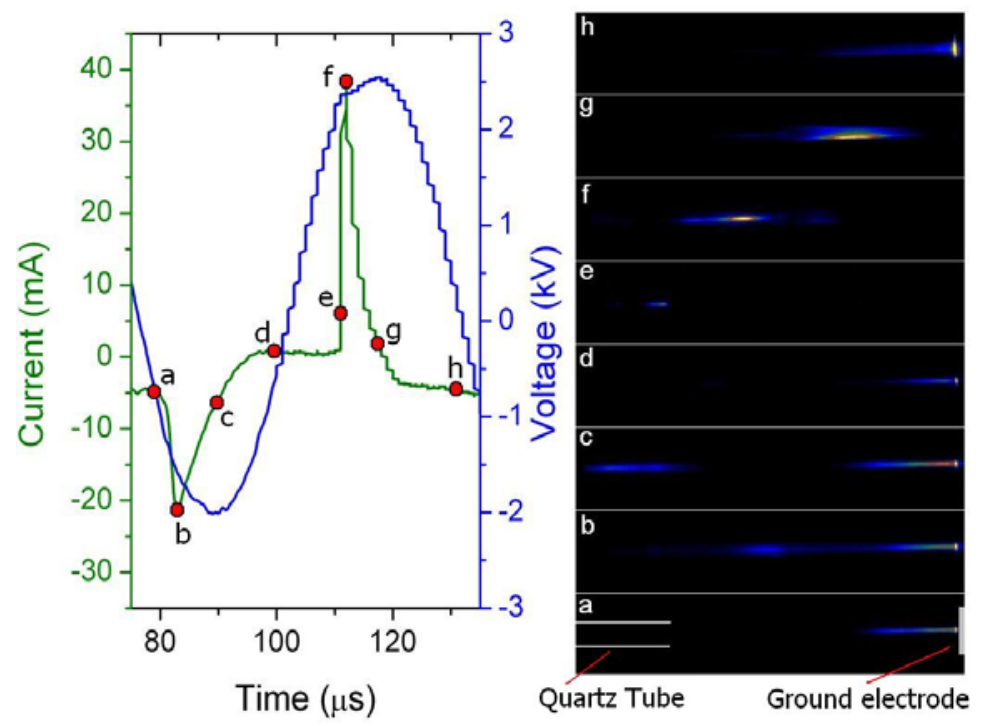

Figure 7: Plasma jet in the bullet mode with the current and voltage traces (left graph) and 10ns exposure images over one complete cycle of the applied voltage (right graph). In (a) of the right graph, the quartz tube is indicated with two horizontal lines on the left and the downstream electrode with a vertical line on the right. 

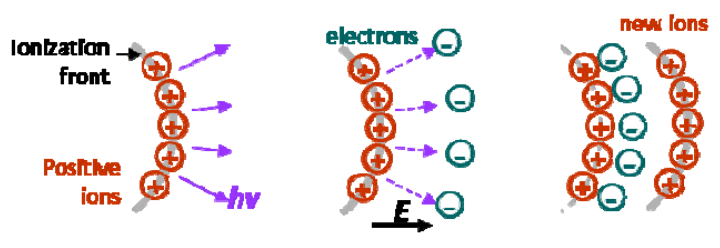

(a) photon production (b) photon lonlzation
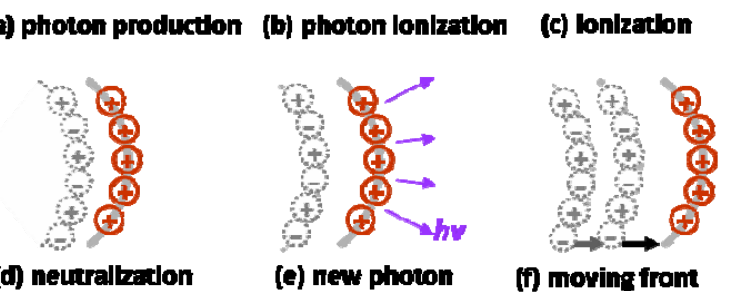

Figure 8: Dawson's photon-ionization theory illustrated, assuming compete neutralization of positive ions by photoelectrons. Instantaneous cathode is on the right. It starts with (a) a positively changed plasma head (position \#1) emitting photons; (b) new electrons produced by photon ionization set up a strong electric field; (c) photoelectrons moving to the original plasma head and leaving behind new positive ions to form the new plasma head (position \#2); (d) ions are completely neutralized; (e) the new plasma head emits photons; (f) this creates a newer plasma head (position \#3) forming a moving plasma head. 


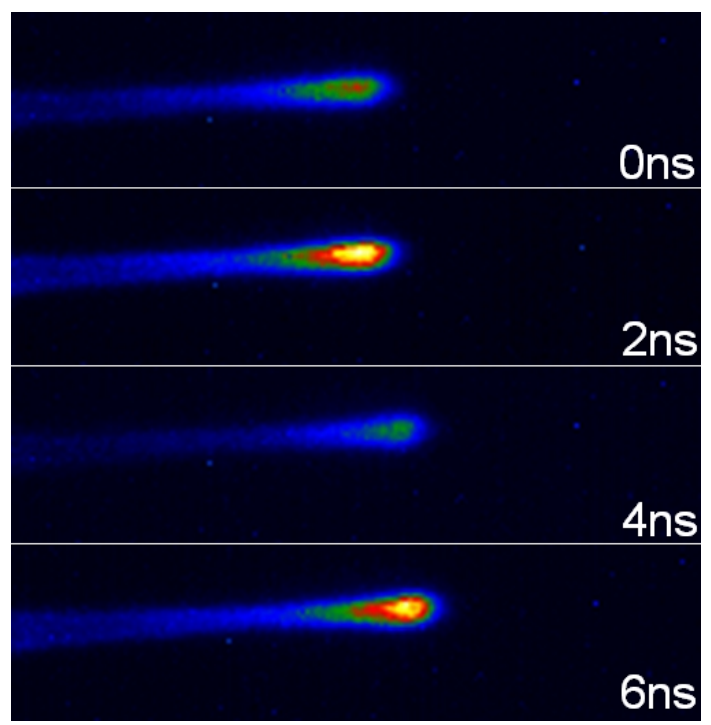

Figure 9: Single-shot 2 ns images of a plasma bullet travelling towards the downstream ground electrode which was the instantaneous cathode. 

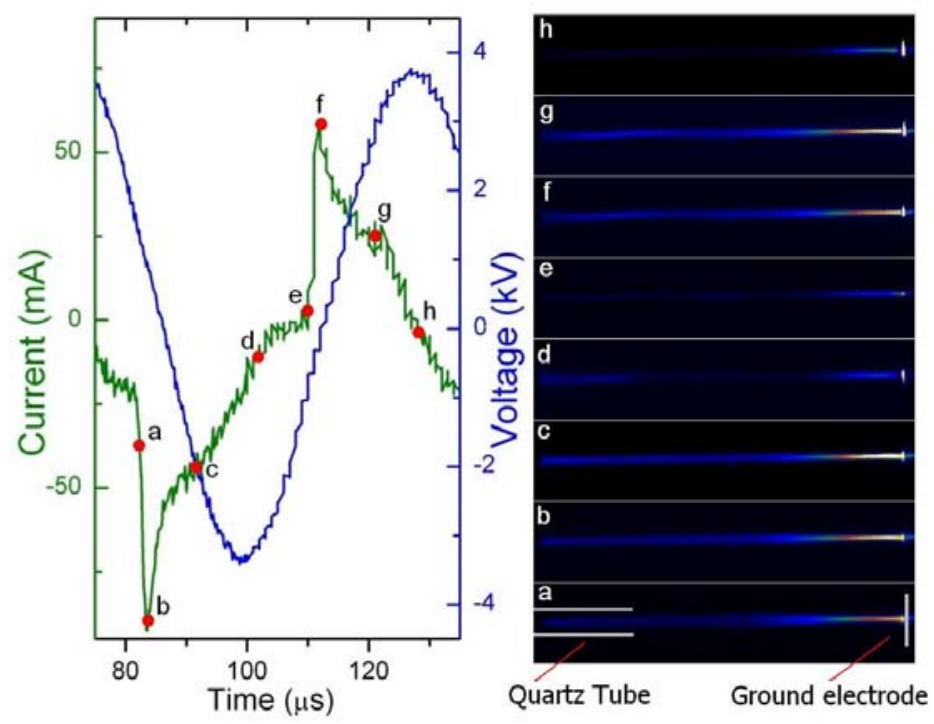

Figure 10: Plasma jet in the continuous mode with the current and voltage traces (left graph) and 10ns exposure images over one complete cycle of the applied voltage (right graph). The quartz tube and the downstream electrode are marked the same way as in figure 7. 


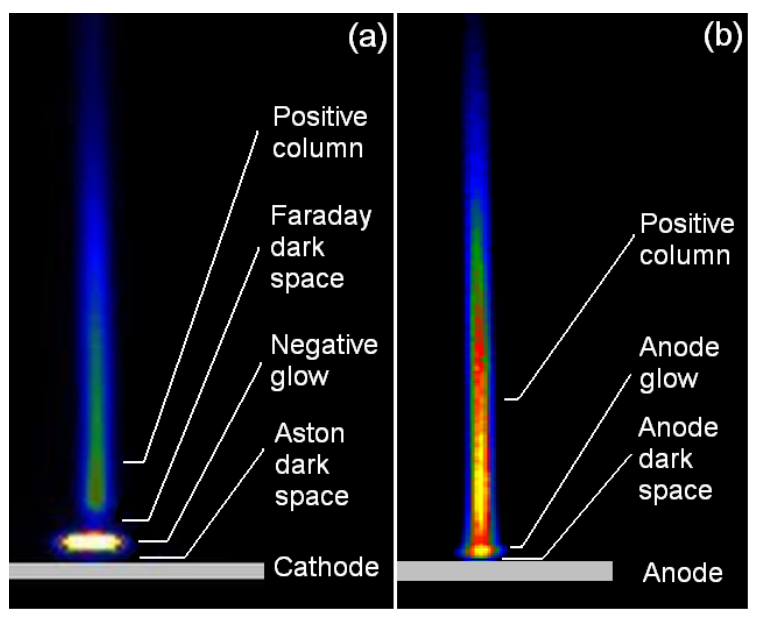

Figure 11: 10ns exposure images showing close-up view of the plasma jet operating in the continuous mode during (a) a positive half cycle and (b) a negative half cycle. 


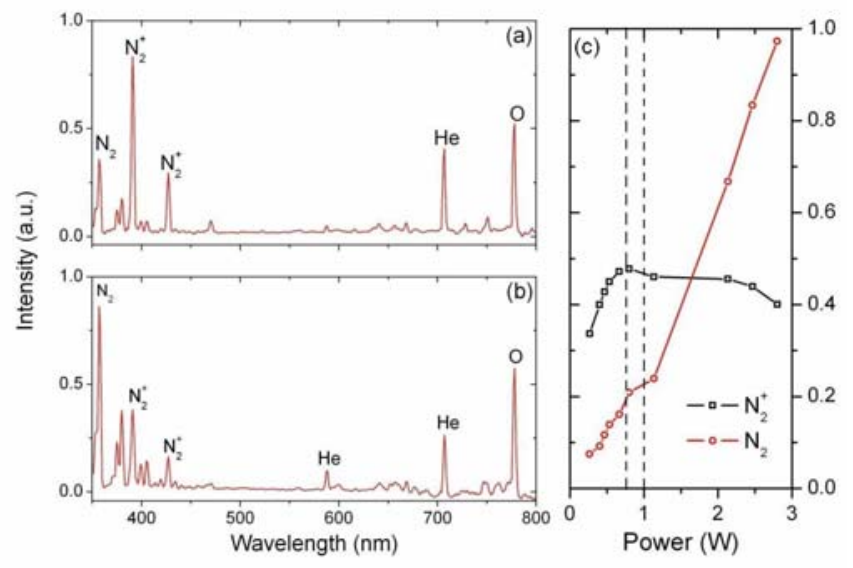

Figure 12: Optical emission spectrum from the atmospheric plasma jet in (a) the bullet mode; (b) the continuous mode; and (c) dissipated power dependence of emission intensities of the excited nitrogen dimmers $(357 \mathrm{~nm})$ and nitrogen dimmer ions (391 nm). The two vertical dashed lines in (c) indicate the transition points from the chaotic to the bullet modes and from the bullet to the continuous modes. 


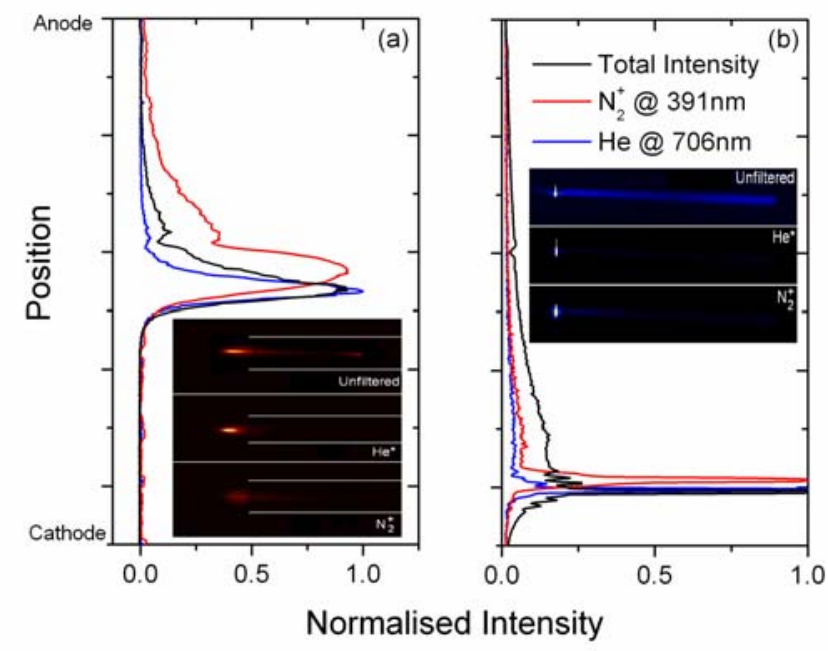

Figure 13: Wavelength filtered intensity profiles for (a) the plasma bullet mode; and (b) the continuous mode. The instantaneous anode is the wrapped electrode and the instantaneous cathode is the downstream ground electrode, in both cases. In the bullet mode, the plasma bullet is moving from the anode to the cathode. 


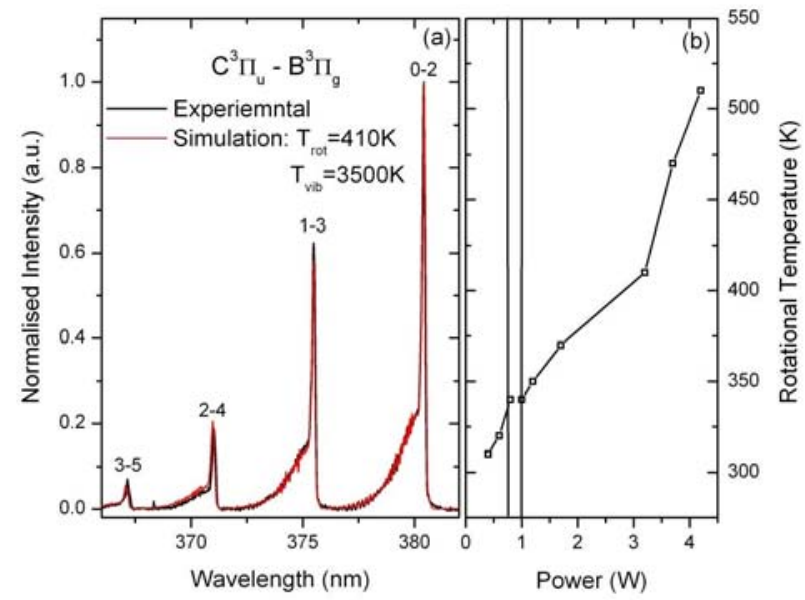

Figure 14: (a) Experimental and simulated nitrogen band data used to determine rotational temperature $\left(T_{\text {rot }}\right)$ and vibrational temperature $\left(T_{\text {vib }}\right)$; (b) dissipated power dependence of the rotational temperature. All measured at the plasma jet contact point on the ground electrode. The two vertical lines in (b) indicate the two mode transition points. 